\title{
Analysis of A Simple Multihop Underwater Acoustic Network
}

\author{
Wenyi Zhang \\ Qualcomm Incorporated \\ Corporate Research and \\ Development \\ 5775 Morehouse Dr. \\ San Diego, CA 92121 \\ wenyizha@gmail.com
}

\author{
Milica Stojanovic \\ Massachusetts Institute of \\ Technology \\ Cambridge, MA 02139 \\ millitsa@mit.edu
}

\author{
Urbashi Mitra \\ Ming Hsieh Department of \\ Electric Engineering \\ University of Southern \\ California \\ Los Angeles, CA 90089 \\ ubli@usc.edu
}

\begin{abstract}
A multihop underwater acoustic network, which consists of a series of equal-distance hops connected by relay transceivers in a tandem, is considered. Messages are originated as coded packets from a source node at one end, relayed (decoded and re-encoded) sequentially hop by hop, and finally received by a destination node at the other end of the network. Several key characteristics of underwater acoustic channels, namely, frequency-dependent signal attenuation and noise, inter-hop interference, half-duplex constraint, and large propagation delay, are taken into account in the analysis. A simple transmission protocol with spatial reuse is considered, and the transmission schedule is designed to satisfy the half-duplex constraint on relay transceivers in the presence of large propagation delay. To efficiently cope with frequency-dependent channel characteristic and inter-hop interference, the power spectral density function of signaling is analytically optimized in a way analogous to water-filling. Furthermore, the problem of determining the minimum number of hops to support a prespecified rate and reliability with and without a maximum coded packet length constraint is examined. Finally, numerical results are presented to illustrate the analysis.
\end{abstract}

\section{Categories and Subject Descriptors}

H.1.1 [Information Systems]: Systems and information theory

\section{General Terms}

Theory

\section{Keywords}

Interference, multihop network, reliability, spectral shaping, underwater acoustic communication

Permission to make digital or hard copies of all or part of this work for personal or classroom use is granted without fee provided that copies are not made or distributed for profit or commercial advantage and that copies bear this notice and the full citation on the first page. To copy otherwise, to republish, to post on servers or to redistribute to lists, requires prior specific permission and/or a fee.

WUWNet'08, September 15, 2008, San Francisco, CA, USA.

Copyright 2008 ACM xxxx ...\$5.00.

\section{INTRODUCTION}

In underwater acoustic communication systems, both bandwidth and power are severely limited, due to exponential (rather than polynomial) attenuation with propagation distance that is also frequency-dependent. As a consequence, multihop networking, in which a longer distance is divided into multiple hops each with a shorter link distance, offers more favorable bandwidth and path loss conditions, and appears as an attractive solution for providing high-rate services for next-generation underwater acoustic communication. The aim of the paper is to give a preliminary analysis of applying multihop networking in underwater acoustic environments.

To gain insights from the analysis, we take a simple network model, in which several hops, each of an identical distance, are connected in a tandem, and information-bearing data packets originating from a source node at one end of the tandem are forwarded in a sequential manner hop by hop to a destination node at the other end of the tandem. Specifically, a relay acoustic transceiver node is employed at the joint of every two consecutive hops. The relay nodes receive the incoming packets, decode them, and re-transmit them on to the next hop, until the final destination is reached. Such a model, though simple, captures the essential elements in multihop networking, and its analysis reveals several interesting behaviors, as will be shown in the paper.

For multihop networks equipped with full-duplex relay transceivers, and operating over wireline type of links without interference among hops, the network capacity is easily shown (by the standard cut-set bound [1] in information theory) to be the minimum link capacity among hops, and the capacity is straightforwardly achieved by implementing good error-control coding for each hop. As a consequence, a significant portion of the literature on multihop networking has instead focused on the network capacity for noncoding relays; see, e.g., [2] - [6] and references therein. The reliability-delay tradeoff in such type of multihop networks was addressed in [7].

The situation, however, dramatically changes for multihop networks with half-duplex relay transceivers and with interference among hops. Such a situation arises in wireless radio links, or underwater acoustic links, which we consider in the present paper. The interference among hops is due to the broadcasting nature of signal propagation in wireless (either radio or acoustic) medium, and it fundamentally changes the network model from a simple multihop tandem network 
to a general relay network, for which the capacity problem is extremely difficult and still open [8]. It is not the aim of the present paper to solve the general information-theoretic problem, but instead, we consider a particular transmission protocol which explicitly considers features of underwater acoustic channels, and analyze its performance. The considered transmission protocol is similar to that in [9], in which for each time slot, a certain subset of nodes simultaneously transmit in the same frequency band with the same average power constraint. Such a form of spatial reuse increases the spectrum utilization efficiency, while at the potential risk of introducing excess interference among receive nodes. Hence there exists an optimal reuse factor, which we identify in the paper. On the other hand, due to the frequency-dependent SNR characteristic in underwater acoustic links as well as the existence of interference, the optimal spectral shape of the signaling scheme can significantly deviate from both flat and water-filling solutions. In the paper, we also solve the spectral shaping problem.

We further consider the problem of determining the minimum number of hops between two fixed end nodes (source and destination), for supporting a prespecified rate and reliability which is measured in terms of packet error probability. Such a problem is motivated by the fact that because deploying nodes is extremely costly for underwater environments, it is wasteful if more nodes than necessary are used. We find conditions for determining the minimum number of hops, under either the ideal situation of capacity-achieving coding with sufficiently large packet lengths, or the more realistic situation of reliability-rate tradeoff with finite packet lengths. We note that, since for underwater acoustic communication systems the largest delay contribution is from signal propagation due to the low speed of sound (cf. [11]), decreasing the end-to-end coding delay is not our objective in the paper.

The remainder of the paper is organized as follows. In Section 2 we describe the multihop network model and a transmission protocol with spatial reuse. In Section 3 we analyze the impact of spatial reuse and signaling power spectrum density (PSD) on the capacity of multihop network, and provide a general method of evaluating the network capacity. In Section 4 we address the problem of determining the minimum number of hops for supporting prespecified rate and reliability requirements. In Section 5, we present numerical results, which aim to illustrate the analysis of the previous sections, and to provide insights to the typical behavior of multihop underwater acoustic networks. Finally in Section 6 we conclude the paper with conclusions and discussion.

\section{MULTIHOP NETWORK MODEL AND TRANSMISSION PROTOCOL}

In this section, we describe the multihop network model and a transmission protocol with spatial reuse, which we shall analyze in the paper. Nodes are denoted by $\mathcal{N}_{k}, k=$ $0,1, \ldots, K$, among which $\mathcal{N}_{0}$ is the source, $\mathcal{N}_{K}$ is the destination, and the remaining nodes are relays located between $\mathcal{N}_{0}$ and $\mathcal{N}_{K}$. For analytical simplicity, we assume that all nodes are located along a straight line, and that every two adjacent nodes are separated by the same distance $d$. However, we note that the analysis in the paper can be modified to treat multihop line networks with possibly non-uniform hop distances. For the moment, the number of hops $K$ and the hop distance $d$ are treated as separate parameters. In Section 4, we will further fix the source-destination distance such that $d$ inversely proportionally decreases as $K$ increases.

\subsection{Signal-to-Noise Ratio Model}

As a signal propagates and is received by a node, its energy dissipates and it is contaminated by noise. We describe these effects using an attenuation function. For wireless radio links, it is common practice to approximate the attenuation function as $A(d) \propto d^{-\alpha}$, where $\alpha$ is a constant decay factor; see, e.g., [13]. For underwater acoustic links, however, both link distance and signaling frequency have impact on the attenuation function which we thus denote by $A(d, f)$ for link distance $d$ and signaling frequency $f$. Consequently, for a transmitted signal with a sufficiently narrow bandwidth centered around carrier frequency $f$ with unit power, the received signal has a frequency-dependent SNR denoted by $\rho(d, f)$.

In the paper, the analysis is derived for, and thus is applicable to, general relationship of $\rho(d, f)$. For numerical evaluation, we consider a specific attenuation and noise model as described in [12]. The attenuation, or path loss that occurs over a distance $d \mathrm{~km}$, for a narrow-band signal of carrier frequency $f \mathrm{kHz}$, obeys

$$
A(d, f) \propto d^{\kappa} a(f)^{d},
$$

where $\kappa$ is called the spreading factor, which we take as $\kappa=$ 1.5 (a.k.a. practical spreading), and $a(f)$ is the frequencydependent absorption coefficient, illustrated in Figure 1 following [12].

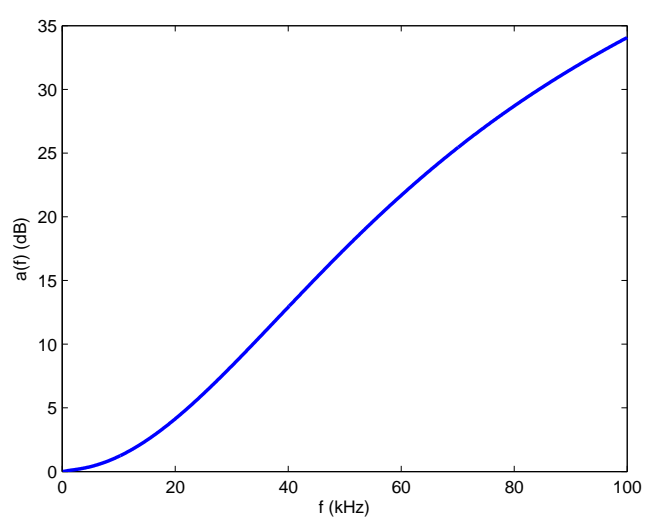

Figure 1: Absorption coefficient $a(f)$ (in dB) versus frequency $f$ (in $\mathbf{k H z}$ ).

Assuming the absence of site-specific noise, the receiver is affected by colored ambient noise, whose PSD is illustrated in Figure 2 following [12].

Jointly affected by the attenuation $A(d, f)$ and the noise PSD $N(f)$, the SNR (density) as a function of narrow-band signal carrier frequency $f$ and receive distance $d$ is

$$
\rho(d, f)=\frac{1}{A(d, f) N(f)} .
$$

As we shall see, the fact that SNR depends upon both distance and frequency has a fundamental impact upon underwater acoustic communication system design. 


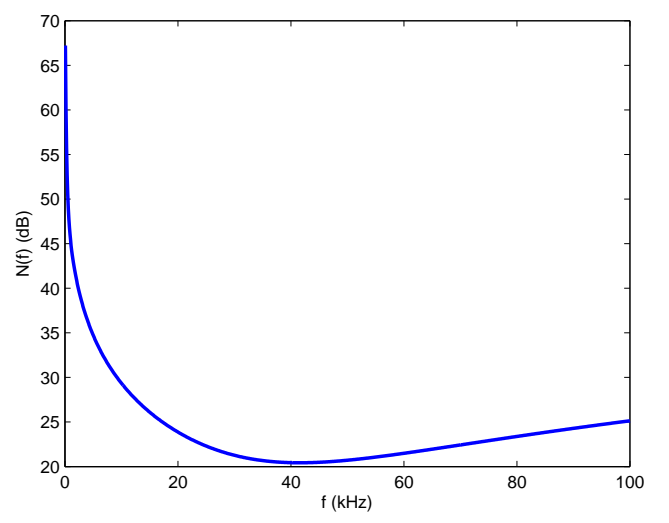

Figure 2: Noise PSD $N(f)$ (in dB) versus frequency $f$ (in $\mathbf{k H z}$ ), with shipping activity factor $s=0.5$ and wind speed $w=0$, corresponding to calm seas and a moderate shipping activity.

\subsection{Transmission Protocol with Spatial Reuse}

Currently, most acoustic modems can only operate in a half-duplex mode. That is, each relay node cannot simultaneously transmit and receive signals. Such a half-duplex constraint thus requires the transmission protocol be properly scheduled to avoid activating both transmit and receive modes of a relay. In the paper, we focus on a specific class of transmission protocols with spatial reuse, such that for each time slot, a certain subset of nodes transmit while another corresponding subset of nodes receive, without violating the half-duplex constraint.

For underwater acoustic communication, the propagation delay of signaling is typically large, and thus needs to be carefully treated in transmission protocols. The speed of sound underwater is approximately $c=1.5 \mathrm{~km} / \mathrm{s}$. Therefore, for typical systems the hop propagation delay can be as large as a fraction of or even several seconds, much larger than packet lengths in many systems. In transmission protocols with spatial reuse, to account for the large propagation delay per hop, it is necessary to adjust the timings of transmit and receive nodes accordingly, as demonstrated by the following example.

In the example, we consider a four-hop network; see Figure 3 . Each hop has distance $d \mathrm{~km}$, thus incuring a propagation delay of $\tau=d / c$ s.

Due to the propagation delay, a data packet sent from time 0 to $T$ will be received by the desired node from time $\tau$ to $(T+\tau)$. To efficiently utilize the time resource, in the example, we consider a slotted model such that each time slot has a duration of $T=\tau / 2$ and each data packet fits exactly into one time slot. In time slot 0 , corresponding to time interval $[0, T=\tau / 2]$, nodes $\mathcal{N}_{0}$ (source) and $\mathcal{N}_{2}$ transmit. However, due to propagation delay, nodes $\mathcal{N}_{1}$ and $\mathcal{N}_{3}$ receive the packets sent by $\mathcal{N}_{0}$ and $\mathcal{N}_{2}$, respectively, in time interval $[\tau=2 T, 3 \tau / 2=3 T]$, corresponding to time slot 2. Therefore, by induction, we observe that the system can operate if we let $\mathcal{N}_{0}$ and $\mathcal{N}_{2}$ transmit, and $\mathcal{N}_{1}$ and $\mathcal{N}_{3}$ receive, in all even time slots; while $\mathcal{N}_{1}$ and $\mathcal{N}_{3}$ transmit, and $\mathcal{N}_{2}$ and $\mathcal{N}_{4}$ receive, in all odd time slots.

In light of the above example, it is obvious that for a $K$ hop network, if we choose the time slot duration and the

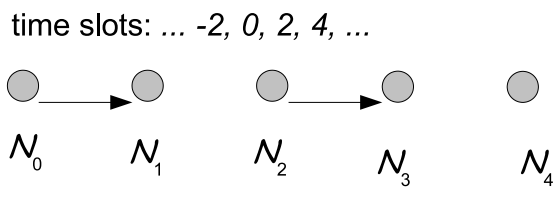

time slots: $\ldots-1,1,3,5, \ldots$

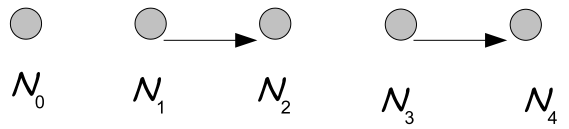

Figure 3: Example: four-hop network with spatial reuse.

length of data packets as

$$
T=\frac{d / c}{Q},
$$

for an arbitrary integer $Q \geq 2$ (in the four-hop example we take $Q=2$ ), then we have the following schedule for continuous transmission:

$$
\begin{aligned}
& \text { time slots: } \ldots,-Q, 0, Q, 2 Q, \ldots \\
& \quad \operatorname{nodes} \mathcal{N}_{0}, \mathcal{N}_{Q}, \mathcal{N}_{2 Q}, \ldots \text { transmit } \\
& \quad \text { nodes } \mathcal{N}_{1}, \mathcal{N}_{Q+1}, \mathcal{N}_{2 Q+1}, \ldots \text { receive } \\
& \text { time slots: } \ldots,-Q+1,1, Q+1,2 Q+1, \ldots \\
& \quad \text { nodes } \mathcal{N}_{1}, \mathcal{N}_{Q+1}, \mathcal{N}_{2 Q+1}, \ldots \text { transmit } \\
& \quad \text { nodes } \mathcal{N}_{2}, \mathcal{N}_{Q+2}, \mathcal{N}_{2 Q+2}, \ldots \text { receive } \\
& \ldots \\
& \text { time } \operatorname{slots:~} \ldots,-1, Q-1,2 Q-1,3 Q-1, \ldots \\
& \quad \operatorname{nodes} \mathcal{N}_{Q-1}, \mathcal{N}_{2 Q-1}, \mathcal{N}_{3 Q-1}, \ldots \text { transmit } \\
& \quad \text { nodes } \mathcal{N}_{Q}, \mathcal{N}_{2 Q}, \mathcal{N}_{3 Q}, \ldots \text { receive }
\end{aligned}
$$

The schedule is also illustrated in Figure 4.

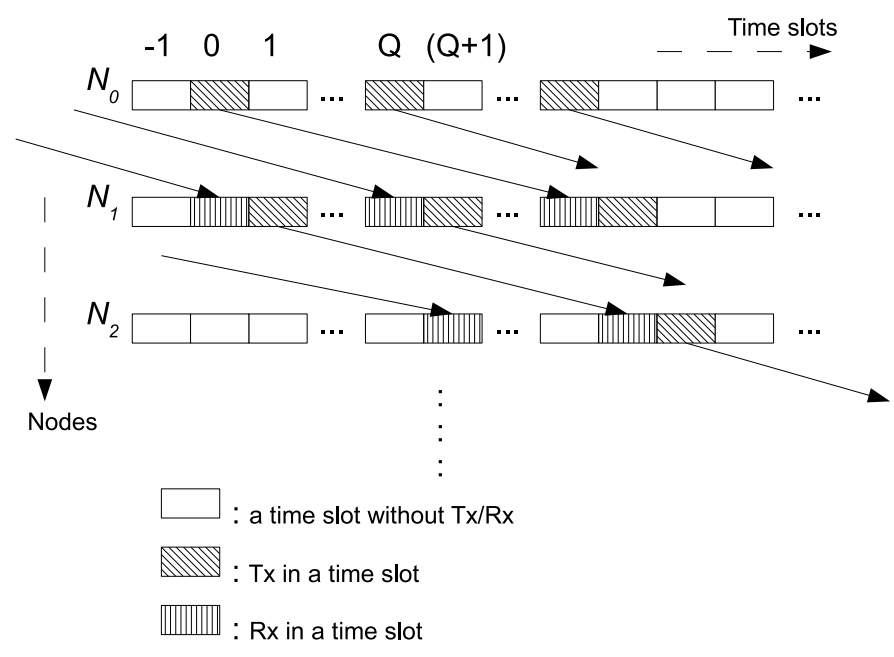

Figure 4: Illustration of the transmission schedule. 


\section{Discussion:}

(1) In the transmission protocol described, only when $Q$ is an integer, all the time slots are fully utilized. In practical systems, there are generally certain losses in time efficiency, due to various non-ideal issues such as unequal hop distances (leading to unequal hop propagation delays), multipath spread (incurring additional propagation delays). Nevertheless, as long as the propagation delay is substantially larger than the data packet length, - a typical situation in practical systems, such losses in time efficiency are negligible.

(2) The transmission schedule in the protocol is just one of the several schedules that achieve full-time transmission without violating the half-duplex constraint. For example, an alternative schedule essentially equivalent to $Q=2$ was considered in [11], under the condition that the ratio between hop propagation delay and time slot length is an odd integer.

In the following sections, we shall focus on the transmission protocol described in this subsection. We call the parameter $Q$ the reuse factor, which plays a key role in performance analysis.

\section{PROTOCOL CAPACITY ANALYSIS AND SIGNALING PSD DESIGN}

In this section, we analyze the impact of spatial reuse and signaling PSD on the transmission rate of a multihop network. In the analysis, we consider Gaussian random coding schemes [1], and let each receive node treat its received signals from nodes other than its immediate upstream node as interference rather than information-bearing coded signals. From an information-theoretic perspective, the coding scheme considered is suboptimal, and in principle (assuming perfect synchronism and coherence among nodes) it is possible to use successive interference cancellation to improve the achievable rate [9]. However, we emphasize that the main purpose of the present paper is to provide insights into practical underwater acoustic networks, and we call the achievable rate the protocol capacity.

Consider the $K$-hop network model. For simplicity, we let all nodes (except the destination $\mathcal{N}_{K}$ since it does not transmit) have an identical average power constraint of $P$. Although unequal power allocation among nodes may lead to certain performance gain, the resulting optimization problem, however, would be non-trivial even for simple wireless radio model without frequency-dependent attenuation [9]. Meanwhile, in order to account for the frequency-dependent link SNR model as described in Section 2.1, transmit nodes may adapt their signaling PSD analogously to water-filling in colored Gaussian noise channels (see, e.g., [1]). Again for simplicity, we let all nodes use the same PSD to shape their transmitted signals.

From the model, it is seen that the bottleneck link is the middle hop from $\mathcal{N}_{\lfloor K / 2\rfloor}$ to $\mathcal{N}_{\lfloor K / 2\rfloor+1}$, since it suffers from the most interference coming from upstream and downstream nodes. To facilitate analysis, we further let the number of hops, $K$, become infinitely large. This is a worst-case scenario in that the interference power is maximized, and the obtained protocol capacity hence serves as a performance lower bound for networks with finite size. For the underwater acoustic link SNR model, our numerical investigation shows that essentially all of the interference is contributed by the two or three nearest interfering nodes, so the infinitenode approximation in fact has a satisfactory accuracy even for networks with a small size.

By inspecting the transmission schedule in Section 2.2, we notice that for a receive node, the interference components in its received signal can only come from earlier transmissions of nodes at the following locations:

$$
\begin{aligned}
& \text { Left: } \quad(Q+1) d,(2 Q+1) d, \ldots,(i Q+1) d, \ldots \\
& \text { away from the receive node; } \\
& \text { and right: }(Q-1) d,(2 Q-1) d, \ldots,(j Q-1) d, \ldots \\
& \text { away from the receive node. }
\end{aligned}
$$

Here it is implicitly assumed that the acoustic modem transmitter is omni-directional.

Consider a narrow bandwidth centered around a carrier frequency $f$, and assume that the signaling of all the nodes is shaped with PSD $S(f)$, we then have that the interferenceto-noise ratio within this narrow bandwidth from all interfering nodes is

$$
\begin{aligned}
I(f) & =\sum_{i=1}^{\infty} \rho((i Q+1) d, f) S(f)+\sum_{j=1}^{\infty} \rho((j Q-1) d, f) S(f) \\
& =\tilde{\rho}_{Q, d}(f) S(f),
\end{aligned}
$$

where

$$
\tilde{\rho}_{Q, d}(f):=\sum_{i=1}^{\infty} \rho((i Q+1) d, f)+\sum_{j=1}^{\infty} \rho((j Q-1) d, f)
$$

characterizes the frequency-dependent interference-to-noise ratio density, parameterized by the reuse factor $Q$ and hop distance $d$.

The signal-to-interference-plus-noise ratio (SINR) at a carrier frequency $f$ hence can be evaluated as

$$
\frac{\rho(d, f) S(f)}{1+\tilde{\rho}_{Q, d}(f) S(f)},
$$

and the information rate achieved by signaling with PSD $S(f)$ is

$$
R_{Q, S(\cdot)}=\frac{1}{Q} \int_{f \geq 0} \log \left(1+\frac{\rho(d, f) S(f)}{1+\tilde{\rho}_{Q, d}(f) S(f)}\right) d f,
$$

where the $1 / Q$ factor is due to the transmission schedule with spatial reuse.

For a given hop distance $d$, we can thus optimize over

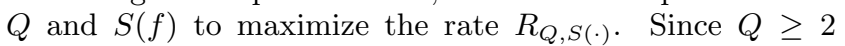
is integer-valued, the optimization over $Q$ can only be performed using discrete search. For a fixed $Q$, however, the optimization over $S(f)$ can be accomplished analytically, as given by the following proposition.

Proposition 1. For a fixed reuse factor $Q$, the signaling $P S D$ that maximizes $R_{Q, S(\cdot)}$ is given by

$$
\begin{aligned}
S(f)= & \frac{1}{2 \tilde{\rho}_{Q, d}(f)\left[\rho(d, f)+\tilde{\rho}_{Q, d}(f)\right]} \cdot\left\{-\left[\rho(d, f)+2 \tilde{\rho}_{Q, d}(f)\right]\right. \\
& \left.+\sqrt{\rho^{2}(d, f)+(4 / \lambda) \rho(d, f) \tilde{\rho}_{Q, d}(f)\left[\rho(d, f)+\tilde{\rho}_{Q, d}(f)\right]}\right\}
\end{aligned}
$$

if $\rho(d, f) \geq \lambda$; and $S(f)=0$ otherwise. The parameter $\lambda>0$ is chosen such that

$$
\int_{f \geq 0} S(f) d f=P
$$

is satisfied. 
Proof: Omitted due to space limitation.

To compute the optimal signaling PSD, we start with a sufficiently small $\lambda>0$, which would lead to $S(f)$ such that $\int_{f>0} S(f) d f>P$, thus violating (7). We then gradually increase $\lambda$, until the equation (7) is satisfied. The corresponding $\lambda$ thus yields the optimal $S(f)$. As we optimize the signaling PSD for every integer $Q \geq 2$, we get the maximum information rate, i.e., the protocol capacity, of the transmission protocols considered in Section 2.2:

$$
C(d)=\max _{Q \geq 2, S(\cdot)} R_{Q, S(\cdot)} .
$$

We use $C(d)$ to explicitly indicate that the protocol capacity is for multihop networks with hop distance $d$.

From the form of (8), it appears that we need to perform an exhaustive search over all integers $Q \geq 2$. In fact, it is not necessary to search over large $Q$ 's. For a fixed $Q$, an immediate upper bound to $R_{Q, S(\cdot)}$ is $(1 / Q) \times$ (the single-hop capacity without interference), which monotonically decreases to zero as $Q$ increases. Therefore, as soon as the upper bound for a certain $Q_{1}$ falls below $\max _{S(\cdot)} R_{Q_{2}, S(\cdot)}$ for any $Q_{2}<Q_{1}$, we can stop searching beyond $Q=Q_{1}$.

\section{MINIMUM NUMBER OF HOPS FOR PRE- SPECIFIED RATE AND RELIABILITY}

The analysis in Section 3 provides a general method of evaluating the information rates of multihop networks with spatial reuse. In this section, we turn to an application of that analysis. Here we fix the end-to-end distance between source and destination to $d_{t} \mathrm{~km}$, and consider how many hops are necessary to support a prescribed rate with a prescribed reliability, measured by an upper bound to packet error probability.

Since packets transmitted through hops are coded, we need a tool to relate reliability and information rate, on basis of a fixed maximum packet length. The following general form of reliability function will be instrumental in analysis of this section:

$$
\begin{aligned}
& E_{\mathcal{C}}(R, T) \text { : packet error probability, with } \\
& R \text { : information rate of the coded packet; } \\
& T \text { : length of the coded packet. }
\end{aligned}
$$

For a specific channel parametrized by a generic parameter $\mathcal{C}$ (for example, SNR in additive white Gaussian noise channels, or crossover probability in binary symmetric channels), a specific coding scheme with information rate $R$, coding block length $T$, and a specific decoding algorithm, $E_{\mathcal{C}}(R, T)$ is the corresponding block error probability. Generally speaking, $E_{\mathcal{C}}(R, T)$ may be obtained either through analytical methods (see, e.g., [14]), or Monte Carlo simulations. In information theory, $E_{\mathcal{C}}(R, T)$ is often interpreted as the minimum block error probability (often its upper and lower bounds) for optimal codes and optimal (maximumlikelihood) decoding algorithms (see, e.g., [15]).

It is not hard to see that for a properly designed coded system, $E_{\mathcal{C}}(R, T)$ should satisfy the following properties:

(a) $E_{\mathcal{C}}(R, T)$ is non-increasing in coding block length $T$;

(b) $E_{\mathcal{C}}(R, T)$ is non-decreasing in information rate $R$;

(c) For rates below a certain threshold (for example, the channel capacity), there exist coding scheme and de- coding algorithm such that $E_{\mathcal{C}}(R, T)$ decreases toward zero as $T \rightarrow \infty$;

(d) For rates above the threshold in (c), $E_{\mathcal{C}}(R, T)$ is always bounded away from zero.

Now let us return back to the problem of determining the minimum number of hops. Consider dividing the length- $d_{t}$ distance between source and destination into $K$ hops, each of which has link distance $d_{t} / K$. Meanwhile, we fix the total power as $P_{t}$, and thus each node, when transmitting, is allocated an average power of $(Q / K) \cdot P_{t}$ (assuming $K \geq Q$ ) if the transmission protocol has a spatial reuse factor of $Q$. From the discussion in Section 3, we have that the protocol capacity $C\left(d_{t} / K\right)$ is given by (8). We assume that the system design requires a prespecified information rate of $R_{0}$, with a prespecified end-to-end packet error probability no greater than $E_{0}$. For many practical applications, $E_{0}$ is a number close to zero, say $10^{-4}$, and the number of hops $K$ is moderate. Therefore, the union bounding technique provides a reasonably accurate reliability constraint for each hop, as $E_{0} / K$. Due to complexity and network-layer considerations, we further assume that the coded packets have a maximum length constraint of $T_{m}$.

We first consider an ideal scenario, where the maximum packet length $T_{m}$ is sufficiently large, such that all rates up until $C\left(d_{t} / K\right)$ can be achieved while satisfying the per-hop reliability constraint $E_{0} / K$. Therefore the number of hops should satisfy

$$
C\left(\frac{d_{t}}{K}\right) \geq R_{0}
$$

and the minimum number of hops immediately follows. The inequality (9) simply asserts that the number of hops should be chosen such that the hop capacity exceeds the prespecified information rate.

We then consider the more realistic scenario where $T_{m}$ is finite. Using the number of hops $K$ to parameterize the hops, we have that it suffices to choose $K$ such that

$$
E_{K}\left(R_{0}, T_{m}\right) \leq \frac{E_{0}}{K},
$$

is satisfied. That is, for each hop, the coding scheme achieves an average packet error probability no greater than $E_{0} / K$, at information rate $R_{0}$, and with packet length $T_{m}$.

\section{NUMERICAL RESULTS}

In this section, we illustrate the analytical results numerically, for the underwater acoustic link model described in Section 2.1.

We take the link parameters as $\kappa=1.5$ (practical spreading), $s=0.5$ (moderate shipping activity), and $w=0$ (calm seas). For convenience, we normalize $\rho(d, f)$ such that $\max _{f} \rho(1 \mathrm{~km}, f)=0 \mathrm{~dB}$. In Figure 5 we plot the SNR density $\rho(d, f)$ as a function of frequency $f$ for $d=0.5,1$ and 2 (in $\mathrm{km}$ ). From Figure 5, we observe that the SNR degradation with distance is quite severe. For each link distance, there is a particular frequency at which the received SNR is maximized, and the resulting maximum SNR decreases rapidly as link distance increases. Furthermore, the SNR vanishes approximately in a dB-linear fashion at high frequencies, and the decay rate grows as link distance increases.

From the SNR characteristic we can evaluate the interferenceto-noise ratio characteristic $\tilde{\rho}_{Q, d}(f)$. We plot $\tilde{\rho}_{Q, d}(f)$ as a 


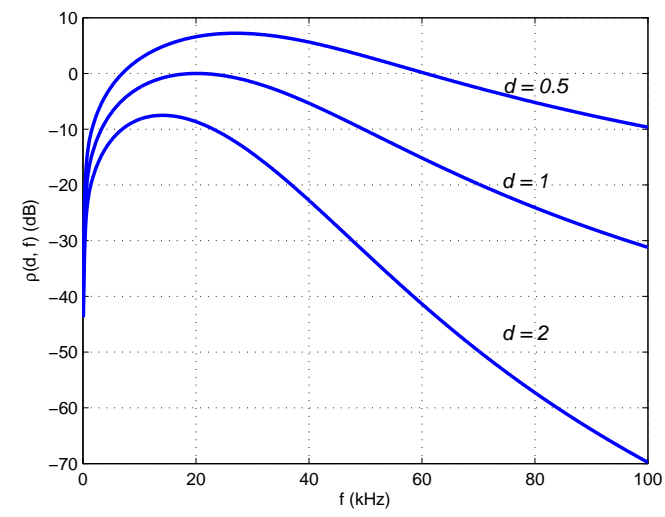

Figure 5: SNR characteristic $\rho(d, f)$ as a function of frequency $f$, for link distances $d=0.5,1$ and 2 (in km).

function of $f$ in Figure 6, for $d=0.5 \mathrm{~km}$, and for spatial reuse factor $Q=2,3,4$ and 5 . We observe that for each

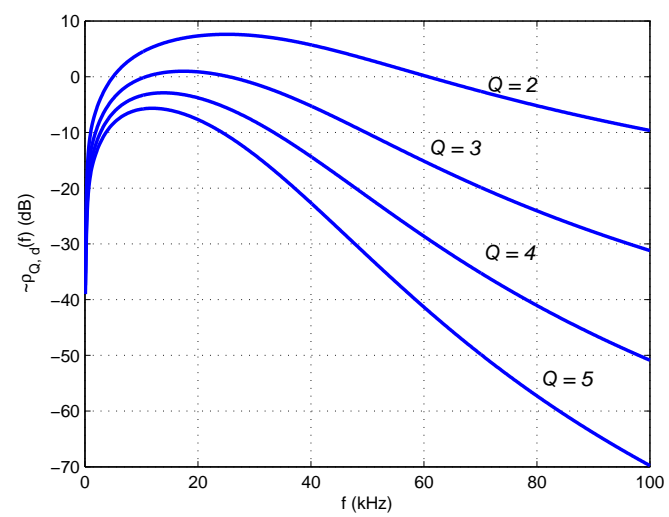

Figure 6: Interference strength $\tilde{\rho}_{Q, d}(f)$ as a function of frequency $f$, for link distances $d=0.5 \mathbf{k m}$, and reuse factor $Q=2,3,4$ and 5 .

$Q$, the $\tilde{\rho}_{Q, d}(f)$ curve closely follows similar trend as $\rho(d, f)$. In fact, our numerical evaluation shows that, most fractions of $\tilde{\rho}_{Q, d}(f)$ are contributed by the nearest interfering node (with distance $(Q-1) d$ away from the receiver), and essentially all of $\tilde{\rho}_{Q, d}(f)$ is due to the two or three nearest interfering nodes. Therefore the similarity between $\tilde{\rho}_{Q, d}(f)$ and $\rho(d, f)$ is not entirely surprising. Such a rapid convergence in $\tilde{\rho}_{Q, d}(f)$ is largely due to the exponential term $a(f)^{d}$ in the attenuation function $A(d, f)$ in (1), and this is a key difference between underwater acoustic channels and wireless radio channels, where interference coming from remote nodes decays slowly. As the reuse factor $Q$ increases, $\tilde{\rho}_{Q, d}(f)$ rapidly decreases, resulting in less interference for the receiving node. However, the $1 / Q$ scaling factor in the achievable rate $R_{Q, S(\cdot)}$ also takes effect. As a consequence, it is usually optimal to operate at $Q=2$ or 3 , as shown in the following figures.

We compute the optimized signaling PSD following Proposition 1 , and plot achievable rates as functions of power $P$ in
Figures 7 and 8 , for hop distance $d=0.5 \mathrm{~km}$ and $2 \mathrm{~km}$, respectively. From both figures, we observe that the optimal

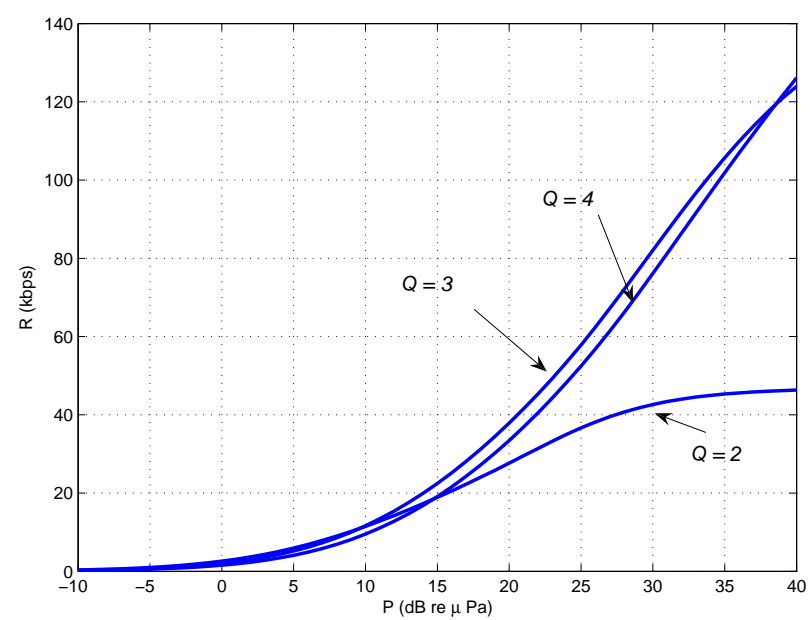

Figure 7: Achievable information rates (optimized over signaling PSD) versus per-node power constraint, for hop distance $d=0.5 \mathrm{~km}$, and reuse factor $Q=2,3$ and 4 .

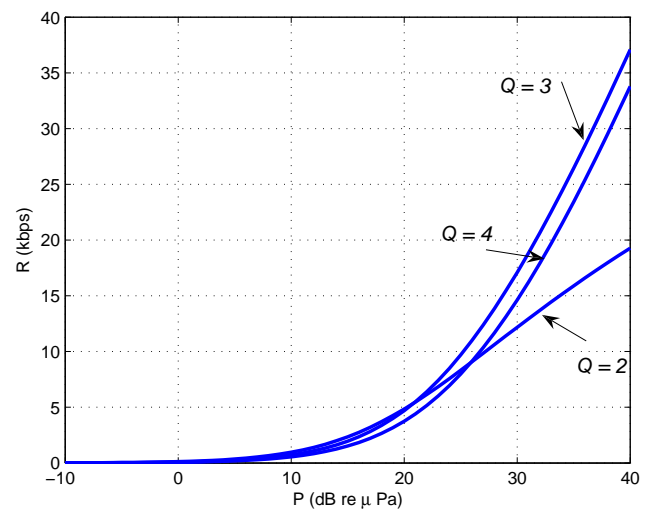

Figure 8: Achievable information rates (optimized over signaling PSD) versus per-node power constraint, for hop distance $d=2 \mathrm{~km}$, and reuse factor $Q=2,3$ and 4 .

reuse factor $Q$ in fact depends on the power constraint $P$. For small $P$, it is optimal to use $Q=2$, while letting $Q=3$ actually does not lead to much rate loss. As $P$ increases, $Q=3$ becomes the optimal choice, and curves for $Q=2$ are in turn outperformed by those for $Q=3$ and 4 .

The optimal signaling PSD as given by Proposition 1 deviates from water-filling, and leads to non-flat receiver SINR versus frequency. We plot the SINR-frequency relationship in Figure 9, for $P=20 \mathrm{~dB}$ re $\mu \mathrm{Pa}, d=1 \mathrm{~km}$, and $Q=2,3$ and 4 . We observe that the SINR curves are quite different for different spatial reuse factors, thus indicating their dependence on the spectral behavior of interference.

Now we proceed to determining the minimum number of hops for supporting prespecified rate and reliability, as an- 


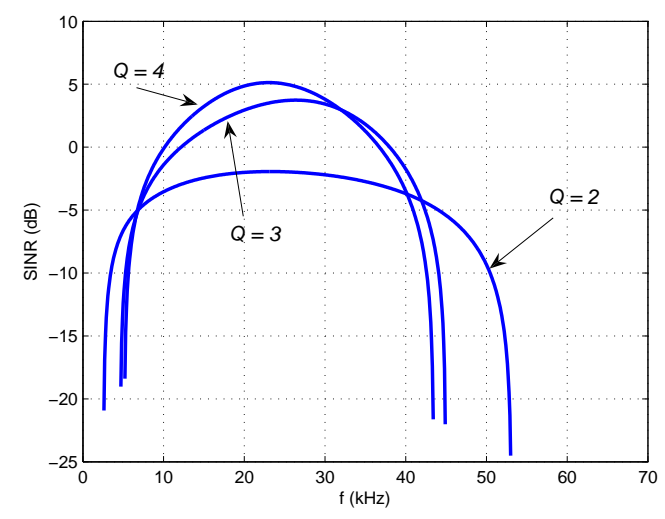

Figure 9: Optimal receiver SINR as a function of $f$, for per-node power constraint $P=20 \mathrm{~dB}$, hop distance $d=1 \mathrm{~km}$, and reuse factor $Q=2,3$ and 4 .

alyzed in Section 4. For the ideal scenario given by (9), we can compute for a sequence of values of $K$ the corresponding protocol capacity of the $K$-hop network $C\left(d_{t} / K\right)$, and find the minimum value of $K$ such that (9) is satisfied. As an example, we plot in Figure 10 the $C\left(d_{t} / K\right)$ versus $K$ curve, for $d_{t}=10 \mathrm{~km}, P_{t}=40 \mathrm{~dB}$ re $\mu \mathrm{Pa}$. We observe that if the prespecified information rate is $R_{0}=75 \mathrm{kbps}$, then the minimum number of hops should be chosen as $K_{m}=15$, implying that the hop distance is $d_{t} / K_{m} \approx 667 \mathrm{~m}$.

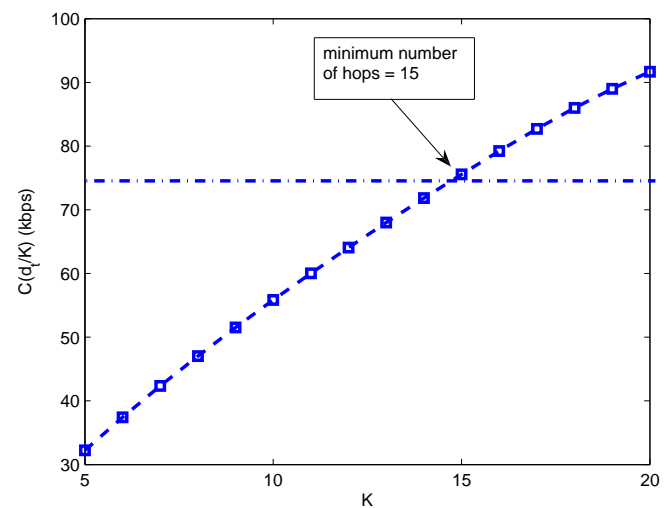

Figure 10: Finding the minimum number of hops necessary to support a prespecified rate. The curve with squares indicates the relationship between the number of hops and the resulting protocol capacity, and the first square strictly above the prespecified rate corresponds to the minimum number of hops, which is $K_{m}=15$ in this plot. System parameters are $d_{t}=10 \mathrm{~km}, P_{t}=40 \mathrm{~dB}$ re $\mu \mathrm{Pa}$, and $R_{0}=75 \mathrm{kbps}$.

Next we consider the realistic scenario, in which both rate and reliability (packet error probability) affect the minimum number of hops, as indicated by (10). Determining the reliability function $E_{K}(R, T)$ is rather tedious, depending upon the particular coding scheme and decoding algorithm used. For simplicity, we evaluate $E_{K}(R, T)$ as the random-coding exponential error bound [15], i.e., $E_{K}(R, T)=\exp [-T$.
$\left.E_{r}(R)\right]$ where $E_{r}(R)$ is the so-called random-coding error exponent, treating the channel SNR characteristic as flat; due to space limitation, we defer the mathematical expressions that we use for numerical evaluation to the full version of the paper.

The minimum number of hops should have the resulting random-coding error exponent satisfy

$$
E_{r}\left(R_{0}\right) \geq \frac{1}{T_{m}} \log \left(\frac{K}{E_{0}}\right) .
$$

We thus plot in Figure 11 two cases: the curve with squares indicates the relationship between $K$ and $E_{r}\left(R_{0}\right)$, and the dashed-dot curve is $\frac{1}{T_{m}} \log \left(K / E_{0}\right)$ versus $K$. System parameters are the same as those of Figure 10, and additionally we assume $E_{0}=10^{-4}$ and $T_{m}=0.1 \mathrm{~s}$. We observe that

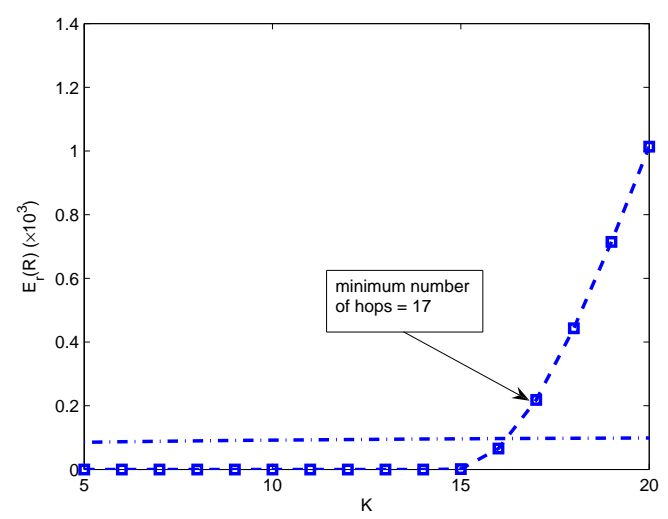

Figure 11: Finding the minimum number of hops necessary to support prespecified rate $\left(R_{0}=75 \mathrm{kbps}\right)$ and packet error probability $\left(E_{0}=10^{-4}\right)$. The curve with squares indicates the relationship between the number of hops $K$ and the resulting random-coding error exponent $E_{r}\left(R_{0}\right)$, and the dashed-dot curve indicates $\frac{1}{T_{m}} \log \left(K / E_{0}\right)$ versus $K$. The first square above the dashed-dot curve corresponds to the minimum number of hops, which is $K_{m}=17$ in this plot. System parameters are $d_{t}=10 \mathrm{~km}, P_{t}=40 \mathrm{~dB}$ re $\mu$ Pa, and $T_{m}=0.1$ s.

as $K$ increases, $E_{r}\left(R_{0}\right)$ initially dwells at zero, then starts increasing, and gradually exceeds $\frac{1}{T_{m}} \log \left(K / E_{0}\right)$. The minimum number of hops turns out to be $K_{m}=17$ (i.e., hop distance $d_{t} / K_{m} \approx 588 \mathrm{~m}$ ), rather than 15 as in the idealized analysis of Figure 10, which overlooked the effect of finite packet length on packet error probability.

Finally, we plot in Figure 12 the minimum number of hops as we change the prespecified information rate from zero to around $80 \mathrm{kbps}$, for the same system parameters as those of Figure 11. The $K_{m}$ versus $R$ relationship is piecewise integer-valued from 1 to around 20, as the information rate increases. Such a plot yields a convenient tool for finding the required number of hops for a given rate. For example, if the prespecified information rate is $R_{0}=75 \mathrm{kbps}$, then inspecting Figure 12 immediately indicates that the minimum number of hops is $K_{m}=17$. 


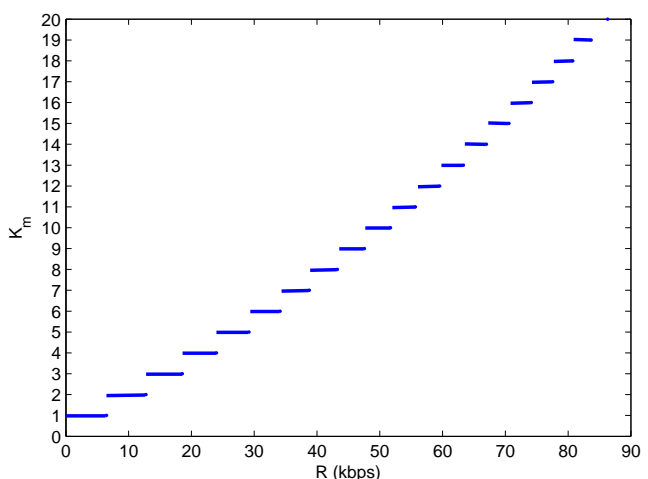

Figure 12: The minimum number of hops as a function of the prespecified information rate $R$ (in kbps). System parameters are $d_{t}=10 \mathrm{~km}, P_{t}=40 \mathrm{~dB}$ re $\mu$ $\mathbf{P a}, E_{0}=10^{-4}$, and $T_{m}=0.1 \mathrm{~s}$.

\section{CONCLUSIONS}

This paper presents a preliminary analysis of multihopping strategies for achieving high-rate transmission in underwater acoustic networking applications. As exemplified in earlier investigations (e.g., [16]), performance of signal detection can be dramatically improved through multihopping in underwater acoustic environments. In this paper, our analysis of information-theoretically achievable rates confirms such a benefit, and yields additional insights into the design of efficient coding schemes. As shown in our numerical study, transmission protocols with a small spatial reuse factor like two or three typically strikes the optimal balance between interference and rate scaling. Furthermore, when inter-hop interference cannot be safely ignored, performing water-filling hop by hop is not optimal and the optimal signaling PSD should be computed taking the interference into account, following Proposition 1. We develop a tool of determining the minimum number of hops for supporting prespecified rate and reliability, and exemplify its application through the random-coding exponential error bound, whereas we note that for practical systems it may be more relevant to evaluate the channel reliability function in an ad hoc fashion for the specific coding scheme used. Our numerical result indicates that, in determining the minimum number of hops, the ideal assumption of infinitely large packet length may yield overly optimistic estimate of the minimum number of hops, and it is usually necessary to take into account the coding block length versus decoding error probability tradeoff revealed by the channel reliability function.

\section{Acknowledgments}

The work of W. Zhang and U. Mitra was supported in part by NSF OCE-0520324; and the work of M. Stojanovic was supported in part by ONR MURI N00014-07-1-0738, NOAA Sea Grant NA060AR4170019, NSF 0520075 and 0427502.

\section{REFERENCES}

[1] T. M. Cover and J. A. Thomas, Elements of Information Theory, John Wiley \& Sons, Inc., New York, 1991.

[2] C. A. Desoer, Communication Through Channels in Cascade, Ph.D. dissertation, Massachusetts Institute of Technology, Cambridge, MA, 1953.

[3] R. A. Silverman, "On Binary Channels and Their Cascades," IRE Trans. Inform. Theory, Dec. 1955, pp. 19-27.

[4] E. C. Posner and A. L. Rubin, "The Capacity of Digital Links in Tandem," IEEE Trans. Inform. Theory, May 1984, pp. 464-470.

[5] A. B. Kiely and J. T. Coffey, "On the Capacity of a Cascade of Channels," IEEE Trans. Inform. Theory, Jul. 1993, pp. 1310-1321.

[6] U. Niesen, C. Fragouli, and D. Tuninetti, "Scaling Laws for Line Networks: From Zero-Error to Min-Cut Capacity," in Proc. IEEE International Symposium on Information Theory (ISIT), Seattle, Jul. 2006.

[7] W. Zhang and U. Mitra, "Multihopping Strategies: An Error-Exponent Comparison," in Proc. IEEE International Symposium on Information Theory (ISIT), Nice, France, Jun. 2007.

[8] T. M. Cover and A. A. El Gamal, "Capacity Theorems for the Relay Channel," IEEE Trans. Inform. Theory, Vol. 25, No. 5, pp. 572-584, Sep. 1979.

[9] M. Sikora, J. N. Laneman, M. Haenggi, D. J. Costello, Jr., T. E. Fuja, "Bandwidth- and Power-Efficient Routing in Linear Wireless Networks," IEEE Trans. Inform. Theory, Vol. 52, No. 6, pp. 2624-2633, Jun. 2006.

[10] M. Stojanovic, "Capacity of a Relay Acoustic Channel," in Proc. the MTS/IEEE Oceans 2007, Oct. 2007, Vancouver, BC, Canada.

[11] W. Zhang and U. Mitra, "A Delay-Reliability Analysis for Multihop Underwater Acoustic Communication," in Proc. the Second ACM International Workshop on Underwater Networks (WUWNet), Sep. 2007, Montreal, Quebec, Canada.

[12] M. Stojanovic, "On the Relationship Between Capacity and Distance in an Underwater Acoustic Communication Channel," in Proc. the First ACM International Workshop on Underwater Networks (WUWNet), Sep. 2006, Los Angeles, CA, USA.

[13] T. S. Rappaport, Wireless Communications: Principles and Practice, Prentice Hall, 2002.

[14] S. Lin and D. J. Costello, Error Control Coding, Prentice Hall, 2004.

[15] R. G. Gallager, Information Theory and Reliable Communication, New York: Wiley, 1968.

[16] C. Carbonelli and U. Mitra, "Cooperative Multihop Communication for Underwater Acoustic Networks," in Proc. the First ACM International Workshop on Underwater Networks (WUWNet), Sep. 2006, Los Angeles, CA, USA. 